\title{
An UXor among FUors: extinction-related brightness variations of the young eruptive star V582 Aur
}

\author{
P. Ábrahám ${ }^{1}$, Á. Kóspál ${ }^{1,2}$, M. Kun ${ }^{1}$, O. Fehér ${ }^{1}$, G. Zsidi ${ }^{1,3}$, \\ J.A. Acosta-Pulido ${ }^{4,5}$ \\ ${ }^{1}$ Konkoly Observatory, Research Centre for Astronomy and Earth Sciences, Hungarian \\ Academy of Sciences, Konkoly-Thege Miklós út 15-17, H-1121, Budapest, Hungary \\ ${ }^{2}$ Max Planck Institute for Astronomy, Königstuhl 17, D-69117 Heidelberg, Germany \\ ${ }^{3}$ Eötvös Loránd University, Dept. Astronomy, Pázmány Péter sétány 1/A, H-1117 Budapest \\ ${ }^{4}$ Instituto de Astrofísica de Canarias, Avenida Vía Láctea, E-38205 La Laguna, Tenerife, Spain \\ ${ }^{5}$ Departamento de Astrofísica, Universidad de La Laguna, E-38205 La Laguna, Tenerife, Spain \\ email: abraham@konkoly.hu
}

\begin{abstract}
The early evolution of Sun-like stars may be interspersed by energetic FU Orionis (FUor) type accretion outbursts. We analysed eight years of photometric and spectroscopic variability of V582 Aur, a bona fide FUor, in outburst. While the accretion rate derived from nearinfrared measurements was constant, radical brightness changes occurred due to dust clumps crossing the line of sight. The brightness minima resemble the variability patterns of the UXor phenomenon. Orbiting density enhancements or short-lived clumps moving in and out of the line-of-sight may explain these observations. Our message is that during FUor outbursts the inner disk is a dynamically active place, affecting the initial conditions for planet formation.
\end{abstract}

Keywords. accretion disks, stars: pre-main-sequence, circumstellar matter

\section{Our target}

V582 Aur is a pre-main-sequence star that brightened by 4-5 magnitudes in 1986, and is still in its high state. The brightening turned out to be an FU Orionis-type outburst, when mass accretion from the inner disk onto the protostar increases by 100-1000× for decades, due to a still debated physical instability (Hartmann \& Kenyon 1996). According to Gaia DR2, the distance of V582 Aur is $2.4_{-0.4}^{+0.7} \mathrm{kpc}$ (Bailer-Jones et al. 2018), which implies that the outburst luminosity is $500-1050 \mathrm{~L}_{\odot}$, powerful enough to modify the density structure, mineralogy, and chemistry of the inner disk. Changes in both the accretion rate and the disk structure are expected to lead to optical-infrared variations, that has indeed been observed in V582 Aur. Here we analyze its multiwavelength light curves to learn about the physical processes in the inner disk during a FUor eruption.

\section{Time evolution of the outburst}

Multiepoch optical and infrared observations of V582 Aur between 2010 and 2018 are plotted in Fig. 1. The figure outlines a relatively well defined constant high flux level of the star, that is modulated by two deep minima, in 2012 and in 2016-18. In the first event the system recovered within a year, but the second dip is still ongoing. The source brightened until 2018 February, then entered a new fading period, but new data from 2018 autumn show a higher flux again. The mid-infrared flux evolution is not correlated with the optical-near-IR light curves. 


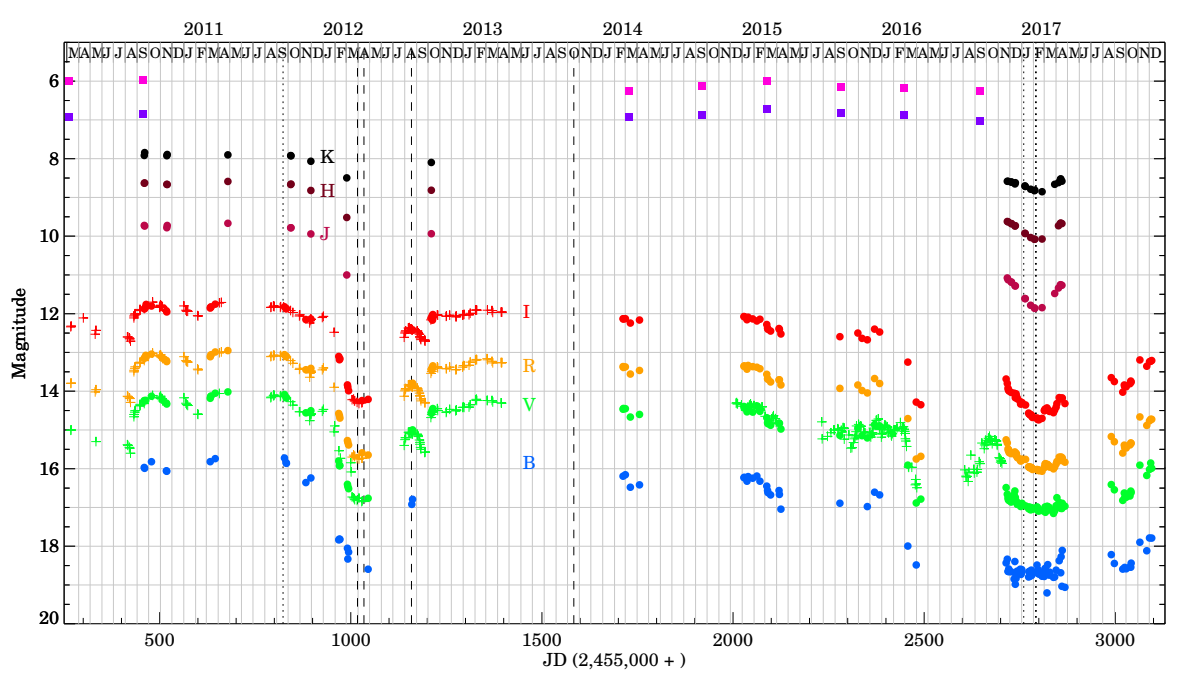

Figure 1. Light curves of V582 Aur. Filled dots are from this work, obtained with the following telescopes: Schmidt \& RCC at Konkoly Observatory, IAC-80 and TCS at Teide Observatory, and the WHT on La Palma. Plus signs are from Semkov et al. (2013), and from ASAS-SN (Shappee et al. 2014, Kochanek et al. 2017). Mid-IR data points are from WISE (filled squares).

\section{Accretion or extinction?}

We modeled the near-IR spectral energy distribution at several epochs by assuming that the emission of the FUor outburst is dominated by a steady, optically thick, and geometrically thin viscous accretion disk, and fitted the circumstellar reddening and accretion rate. We found that while the accretion was relatively constant over the whole period, the extinction increased during the brightness minima. These results imply that the observed variability was caused by extinction changes (Ábrahám et al. 2018).

\section{Nature of the obscuring material}

The two deep minima in 2012 and 2016-18 may suggest an orbiting dust clump periodically occulting the inner part of the system. The different lengths and profiles of the two minima might indicate a viscous spreading of the dust particles along the orbit. With an orbital radius of $2.8 \mathrm{au}$, extinction of $\Delta A_{V}=7.3 \mathrm{mag}$, and dust mass of $\sim 0.004 \mathrm{M}_{\oplus}$, it would be a minor density fluctuation within the disk. Alternatively, dust could be freshly created by colliding planetesimals, or be part of a giant ( $\mathrm{M} \sim 2 \times 10^{6}$ Hale-Bopp) exocomet visiting the inner system on an excentric orbit. The mid-infrared flux peak in 2015 (Fig. 1) may mark the periastron of the exocomet's orbit (Ábrahám et al. 2018).

Acknowledgements. This project has received funding from the European Research Council (ERC) under the European Union's Horizon 2020 research and innovation programme under grant agreement No 716155 (SACCRED).

\section{References}

Ábrahám, P., Kóspál, Á., Kun, M. et al. 2018, ApJ, 853, 28

Bailer-Jones, C.A.L., Rybizki, J., Fouesneau, M., Mantelet, G., Andrae, R. 2018, AJ, 156, 58

Hartmann, L., \& Kenyon, S.J. 1996, ARAA, 34, 207

Kochanek, C.S., Shappee, B.J., Stanek, K.Z. et al. 2017, PASP, 129, 104502

Semkov, E.H., Peneva, S.P., Munari, U. et al. 2013, A\&A, 556, A60

Shappee, B.J., Prieto, J.L., Grupe, D. et al. 2014, ApJ, 788, 48 